\title{
Vitamin D Deficiency in the Elderly Patients and Role of Vitamin D in the Prevention of COVID-19 Infection
}

\author{
Nauman Khan and Tahmina
}

\section{ABSTRACT}

SARS Covid'19 is declared as a global pandemic by World Health Organization [1]. The aim of this short report is to find out the association of Vitamin D in prevention of Covid'19 in elderly patients who are already having other co-morbidities. People of any age are susceptible to Corona virus but the elderly patients and patients with chronic underlying diseases are at higher risk. Many elderly patients with COVID-19 are at risk of malnutrition [2].

\begin{abstract}
Vitamins are of two types, Lipid soluble vitamins and water-soluble vitamins [3]. Lipid soluble vitamins can be stored in the body while water soluble vitamins cannot be stored in the body, hence needed to take regularly [4]. Vitamin D is a lipid soluble vitamin also it can be synthesized by our own body.
\end{abstract}

Keywords: SARS Covid-19, Malnutrition, Risk factors, 1,25dihydroxyvitamin D, Frailty, mortality, morbidity.

\section{INTRODUCTION}

Macronutrients and Micronutrients have significant importance in human life for maintaining the physiological functions. Along with Macronutrients to be taken in a recommended amount, the micronutrients should also be consumed adequately. Micronutrients consist of vitamins (fat soluble and water soluble) and minerals. Supplementation is also a way to meet the required daily amounts of micronutrients for individuals following a special lifestyle.

\section{A. Vitamin D and Its Function}

Vitamin D is also known as calciferol,1,25-dihydroxy vitamin D (calcitriol), vitamin D3 or cholecalciferol, vitamin D2 or ergocalciferol. Once the vitamin enters the body it needs to get activated. Activation occurs through the action of the liver and the kidneys [5].

Vitamin D can be synthesized in the body. It has distinct target organs. Those people who are not exposed to sunlight properly, or there might be impaired absorption of vitamin D deficiency or any abnormality in vitamin D activation can cause vitamin D deficiency. In children, rickets can occur while in adults or elderly people they can face osteomalacia. The dietary vitamin $\mathrm{D}$ is absorbed in the duodenum along with lipids then it is transported to the liver through chylomicrons.

Vitamin D plays an important role as a hormone in the regulation of phosphorus and calcium metabolism. Also, it maintains the normal plasma level of calcium and phosphorus. In osteomalacia serum calcium is decreased, lineage [6].
Submitted: March 10, 2021

Published: March 31, 2021

ISSN: 2593-8339

DOI: $10.24018 /$ ejmed.2021.3.2.765

Dr. Nauman Khan

MBBS, MRCP(UK), MRCPE

Consultant Geriatrician

Southport and Ormskirk Hospital NHS

Trust, UK.

(e-mail: nauman.khan@nhs.net )

Tahmina

Clinical Dietitian

BS Nutrition

Dow University of Health Sciences,

Karachi, Pakistan.

(e-mail: tehminaimtiaz145@gmail.com)

mineralization is insufficient, bones become soft and pliable, and bone becomes deformed.

\section{B. Effect of Vitamin D on Bone Turnover}

Bone is a living tissue, which makes up the body skeleton and is one of the hardest structures of the animal body. It serves as a storage site for minerals. In bones continuous remodeling take place through its anabolic effect which is known as osteoblast and catabolic effect which is known as osteoclast. Osteoblast is mononucleated cells responsible for the synthesis and secretion of the macromolecular organic constituents of bone matrix. They are derived from osteoprogenitor cells of mesenchymal origin, which are present in the bone marrow and other connective tissues. Its function is to form new bone via synthesis of various proteins and polysaccharide and regulation of bone remodeling and mineral metabolism. Osteoclasts are bone resorbing cells derived from hematopoetic cells of monocyte-macrophage

\section{COVID-19 and Elderly Patients}

A novel coronavirus has been originated in Wuhan, China and now has been spread in many different countries throughout the globe. People of any age are susceptible to Corona virus but the elderly patients and patients with chronic underlying diseases are at higher risk. COVID-19 associated mortality and morbidity increase with age. Overall, $45 \%$ of hospitalizations, $53 \%$ of intensive care unit (ICU) admissions, and $80 \%$ of deaths associated with COVID-19 were among the elderly. Serum $25(\mathrm{OH}) \mathrm{D}$ levels is the best index of vitamin D nutritional status. Hypovitaminosis D is highly prevalent in the elderly because of the impaired ability 
to synthetized vitamin D by the skin, limited sun exposure and malabsorption [7].

\section{DISCUSSION}

Older adults are at increased risk of vitamin D deficiency [8]. Those older adults who already are vitamin D deficient when get corona virus disease they get worse affected by the morbidity outcomes.

As a result of growing existence expectancy \& sedentary lifestyle, osteoporosis is rising as a primary trouble for postmenopausal females and aged males. The recent National Osteoporosis Risk Assessment study has shown that nearly half of American women aged 50 or older have undetected low bone mass and the lifetime risk of fracture is approximately $40 \%$ for white Caucasian women and $13 \%$ for white men. It is projected that more than about $50 \%$ of all osteoporotic hip fractures will occur in Asia by the year 2050.

Those elderly people who live in moderate climate countries consume diet very low in vitamin D rich sources they have low amount of 7 dehydrocholesterol due to which skin synthesis becomes lower. In winter and spring due to climate change and low sunlight, the concentration of $25(\mathrm{OH})$ D3 decreases. The exposure to sunlight is more strongly affects the elderly males rather than females. Other factors that are responsible for low vitamin D levels are poor environmental circumstances, inadequate physical activity, low socioeconomic background, drug usage, overall poor health and malnutrition [10].

Supplementation can be given to make vitamin D levels in a moderate range. Gastrointestinal diseases can lead to malabsorption of cholecalciferol and ergocalciferol and it interacts with their transformation in the liver. Vitamin D deficiency can also lead to metabolic syndrome such as cardiovascular diseases, depression, T2DM and cancers. Frailty refers to a loss of physiologic reserve that makes a person susceptible to disability from minor stresses. Frail elderly people are severely disabled. Some common features of frailty are weakness, muscle wasting, frequent falls, weight loss, immobility, incontinence, exercise intolerance, instability of chronic diseases. Some other associated features of frailty include older age, gender, educational level, socioeconomic background, and poor health. Healthy diet with all macronutrients and micronutrients in a balanced amount can help to lower the risk of frailty. Supplements are also helpful to compensate with low nutritive value of any micro or macro nutrients. However, an adequate physical activity can also lead to a healthier life.

\section{METHOD}

A systemic search was done using published literature about vitamin D deficiency and ADHD. Articles about Risk factors of Vitamin D deficiency and its symptoms were referred. However, they were narrowed down to elderly people only. International researches were analyzed more than regional researches because there was very limited published data in regards to our topic from South Asian region. All articles were searched through PubMed and Google Scholar.

\section{CONCLUSION}

Due to the shortage of information, symptomatic management is employed for COVID-19 infection together with chemical element medical aid and mechanical ventilation for those with severe condition. Considering the vary of helpful effects ascribed to viosterol, it is safety and simple administration, moreover as direct effects of viosterol on immune cell proliferation and activity, pulmonic ACE2 expression and reducing physical phenomenon, analysis of viosterol supplementation as associate adjuvant therapeutic intervention may well be of considerable clinical and economic significance.

In conclusion, given the high prevalence of vitamin D deficiency and so as to speedily, safely, and considerably raise body fluid concentrations, high-dose is recommended to decrease the risk of COVID-19 severity and mortality. It may be a safe and noninvasive treatment. Patients would take massive doses of vitamin D per week, followed by 1000-1200 IU/d. This may give a fast and proper restoration of body fluid levels, thus, probably triggering associate degree improvement in clinical standing and prognosis. However, prospective clinical studies are needed to deal with this speculation to overcome the obstacles in our current understanding of vitamin $\mathrm{D}$ role as an adjuvant medical care in patients with Corona virus disease [11].

\section{REFERENCES}

[1] nfwp-static.s3.amazonaws.com.

[2] Andrius Bleizgys. "Vitamin D AND COVID-19: It is time to act", International Journal of Clinical Practice, 2020.

[3] www.answers.com.

[4] www.nutritional-supplements-guide.com

[5] -Xudong Mao, Bin Hu, Zhiwen Zhou, Xubin Xing, Yan Wu, Jing Gao, Yue He, Ying Hu, Qihong Cheng, Qing Gong Vitamin D levels correlate with lymphocyte subsets in elderly patients with age-related diseases", Scientific Reports, 2018.

[6] link.springer.com.

[7] https://www.cdc.gov/coronavirus/2019-ncov/index.html.

[8] toppodcast.com.

[9] https://www.slideshare.net/RajeshBendre/vitamin-d-bone-health.

[10] worldwidescience.org.

[11] www.nature.com. 